\section{遶 IUCrData}

ISSN 2414-3146

Received 26 March 2016

Accepted 8 April 2016

Edited by W. T. A. Harrison, University of Aberdeen, Scotland

Keywords: crystal structure; imidazole; hydrogen bonding; $\pi-\pi$ interactions.

\section{CCDC reference: 1472955}

Structural data: full structural data are available from iucrdata.iucr.org

\section{(4Z)-2-Phenyl-1-\{(E)-[4-(propan-2-yl)benzylidene]- amino]-4-[(thiophen-2-yl)methylidene]-1H- imidazol-5(4H)-one}

\author{
Kamni, ${ }^{a}$ Vikram D. Singh, ${ }^{a}$ K. N. Subbulakshmi, ${ }^{\text {b }}$ B. Narayana, ${ }^{\text {b }}$ B. K. Sarojini, ${ }^{c}$ \\ Sumati Anthal ${ }^{\mathrm{d}}$ and Rajni Kant ${ }^{\mathrm{d} *}$
}

${ }^{\mathbf{a}}$ School of Physics, Shri Mata Vaishno Devi University, Katra 182 320, J\&K, India, ${ }^{\mathbf{b}}$ Department of Chemistry, Mangalore University, Mangalagangothri 574 199, D.K., Mangalore, India, ' Department of Industrial Chemistry, Mangalore

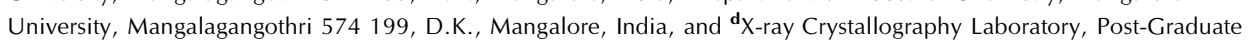
Department of Physics \& Electronics, University of Jammu, Jammu Tawi 180 006, India. *Correspondence e-mail: rkant.ju@gmail.com

In the title molecule, $\mathrm{C}_{24} \mathrm{H}_{21} \mathrm{~N}_{3} \mathrm{OS}$, the imidazole ring subtends dihedral angles of 4.6 (1) and $20.2(1)^{\circ}$ with the thiophene and iso-propylbenzene rings, respectively. The plane of the imidazole ring forms a dihedral angle of $39.9(1)^{\circ}$ with the phenyl ring. An intramolecular $\mathrm{C}-\mathrm{H} \cdots \mathrm{N}$ hydrogen bond closes an $S(6)$ ring. In the crystal, pairs of $\mathrm{C}-\mathrm{H} \cdots \mathrm{O}$ hydrogen bonds link molecules into inversion dimers featuring $R_{2}^{2}(10)$ graph-set motifs. Aromatic $\pi-\pi$ stacking interactions are observed between the thiophene and imidazole rings [centroidcentroid distance $=3.570(2) \AA$ ] and thiophene and benzene rings [centroidcentroid distance $=3.889(2) \AA]$. Weak $\mathrm{C}-\mathrm{H} \cdots \pi$ interactions are also observed.
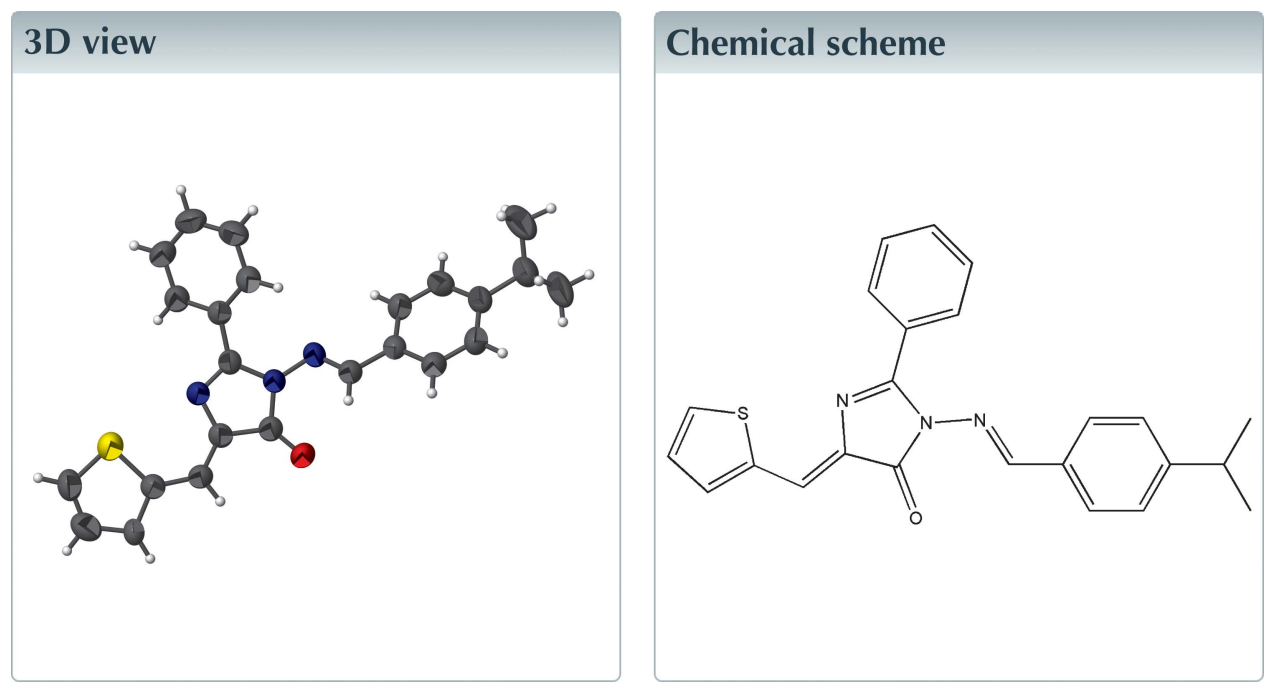

\section{Structure description}

Imidazolones are nitrogen analogues of azlactones bearing an exocyclic double bond at the fourth position, usually called unsaturated 2,4-disubstituted 2-imidazolin-5-ones. Compounds containing imidazolone as well as imine moieties exhibit a range of pharmaceutical activities, such as antimicrobial (Suthakaran et al. 2008; Patel et al. 2006), antioxidant (Suhasini et al.2014) and anticonvulsant activity (Mohamed et al. 2012).

In the title compound (Fig. 1), the imidazole ring subtends dihedral angles of 4.6 (1) and $20.2(1)^{\circ}$ with the thiophene and benzene rings, respectively. The dihedral angle between the phenyl and imidazole rings is $39.9(1)^{\circ}$. The sum of bond angles around N2 is $358.3^{\circ}$, which confirms that the atom N2 is in an $s p^{2}$ hybridized state. The $\mathrm{C} 7=\mathrm{O} 1$ bond distance is 1.218 (5) $\AA$ which is somewhat longer than the standard value for a carbonyl 


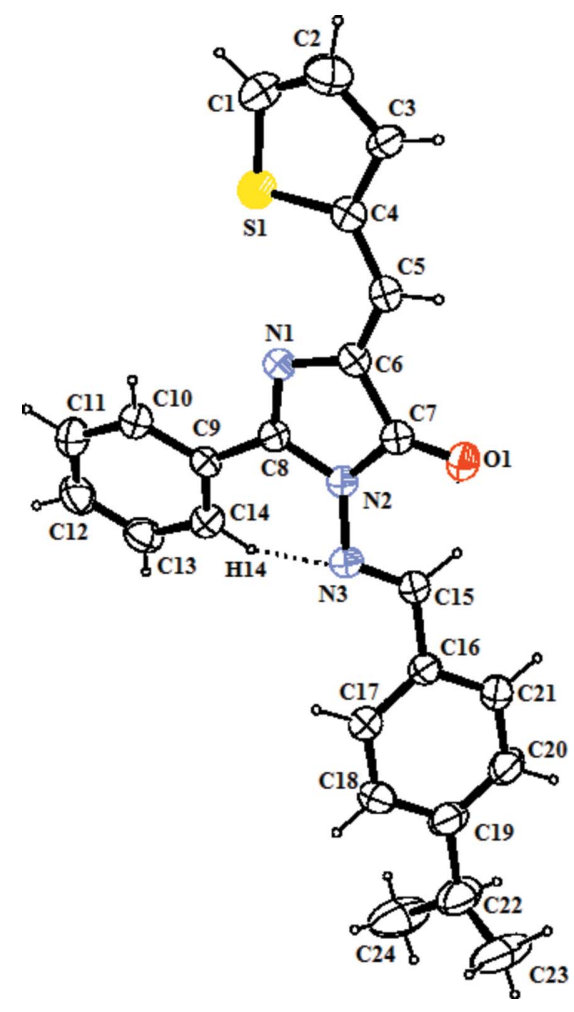

Figure 1

ORTEP view of the title molecule with displacement ellipsoids drawn at the $40 \%$ probability level. Dashed lines indicate the intramolecular hydrogen bond.

group (1.192 $\AA$ ); the lengthening of this double bond may be due to the involvement of this oxygen atom as a hydrogenbond acceptor.

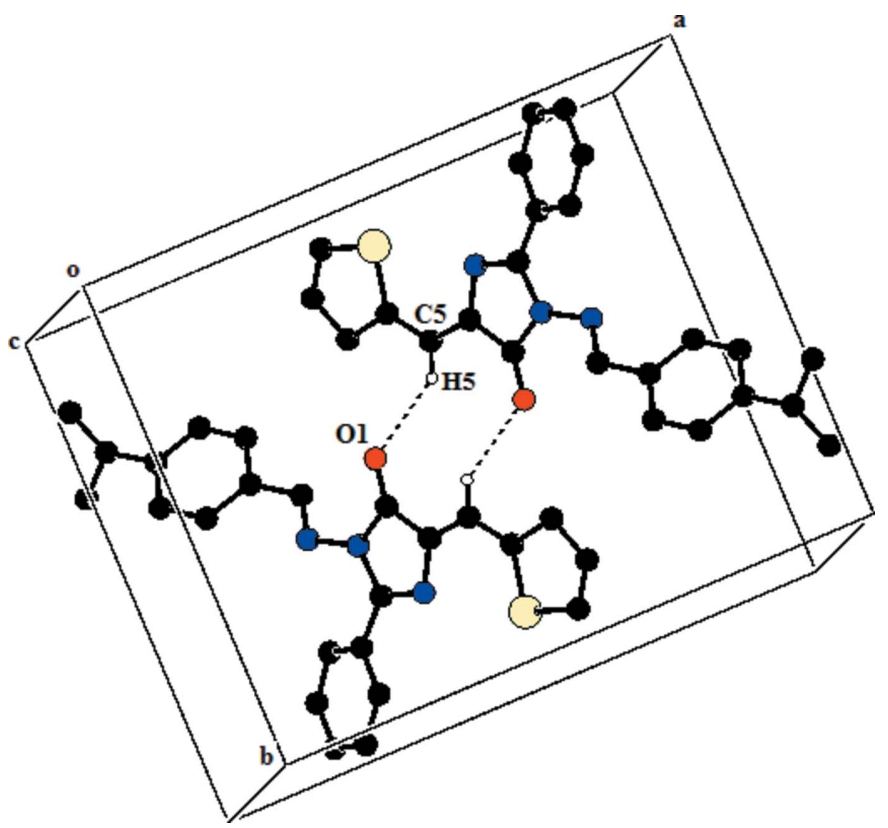

Figure 2

A dimer of molecules of the title compound linked by a pair of $\mathrm{C}-\mathrm{H} \cdots \mathrm{O}$ hydrogen bonds forming an $R_{2}^{2}(10)$ loop.
Table 1

Hydrogen-bond geometry $\left(\AA,^{\circ}\right)$.

\begin{tabular}{lllll}
\hline$D-\mathrm{H} \cdots A$ & $D-\mathrm{H}$ & $\mathrm{H} \cdots A$ & $D \cdots A$ & $D-\mathrm{H} \cdots A$ \\
\hline $\mathrm{C} 14-\mathrm{H} 14 \cdots \mathrm{N} 3$ & 0.93 & 2.38 & $2.960(5)$ & 120 \\
$\mathrm{C} 5-\mathrm{H} 5 \cdots \mathrm{O} 1^{\mathrm{i}}$ & 0.93 & 2.44 & $3.221(5)$ & 142 \\
\hline
\end{tabular}

Symmetry code: (i) $-x+1,-y+2,-z+2$.

In the crystal, centrosymmetric dimeric aggregates (Fig. 2) are formed by pairs of $\mathrm{C}-\mathrm{H} \cdots \mathrm{O}$ hydrogen bonds (Table 1), forming $R_{2}^{2}(10)$ ring motifs. $\pi-\pi$ interactions are observed between the thiophene and imidazole rings [centroid separation $=3.570(2) \AA$, interplanar spacing $=3.528 \AA$ and centroid shift $=0.55 \AA$ ] and the thiophene and benzene rings [centroid separation $=3.889(2) \AA$, interplanar spacing $=2.976 \AA$ and centroid shift $=2.50 \AA]$. Weak $\mathrm{C}-\mathrm{H} \cdots \pi$ interactions are also observed.

\section{Synthesis and crystallization}

A mixture of 3-hydrazinyl-3-oxo-1-(thiophen-2-yl)prop-1-en2-yl]benzamide $(0.01 \mathrm{~mol})$ in 2-propanol $(30 \mathrm{ml})$ with 4(propan-2-yl)benzaldehyde $(0.01 \mathrm{~mol})$ in the presence of one or two drops of sulfuric acid was heated under reflux for $8 \mathrm{~h}$. The reaction mixture was then cooled to room temperature and poured on cold water; the solid mass obtained was

Table 2

Experimental details.

\begin{tabular}{|c|c|}
\hline \multicolumn{2}{|l|}{ Crystal data } \\
\hline Chemical formula & $\mathrm{C}_{24} \mathrm{H}_{21} \mathrm{~N}_{3} \mathrm{OS}$ \\
\hline$M_{\mathrm{r}}$ & 399.50 \\
\hline Crystal system, space group & Monoclinic, $P 2_{1} / c$ \\
\hline Temperature (K) & 293 \\
\hline$a, b, c(\AA)$ & $\begin{array}{l}15.8392(13), 12.9075(10), \\
10.3997(8)\end{array}$ \\
\hline$\beta\left(^{\circ}\right)$ & $103.354(8)$ \\
\hline$V\left(\AA^{3}\right)$ & $2068.7(3)$ \\
\hline$Z$ & 4 \\
\hline Radiation type & Mo $K \alpha$ \\
\hline$\mu\left(\mathrm{mm}^{-1}\right)$ & 0.18 \\
\hline Crystal size (mm) & $0.30 \times 0.20 \times 0.20$ \\
\hline \multicolumn{2}{|l|}{ Data collection } \\
\hline Diffractometer & $\begin{array}{l}\text { Oxford Diffraction Xcalibur, } \\
\text { Sapphire3 }\end{array}$ \\
\hline Absorption correction & $\begin{array}{l}\text { Multi-scan (CrysAlis PRO; Oxford } \\
\text { Diffraction, } 2010\end{array}$ \\
\hline$T_{\min }, T_{\max }$ & $0.636,1.000$ \\
\hline $\begin{array}{l}\text { No. of measured, independent and } \\
\text { observed }[I>2 \sigma(I)] \text { reflections }\end{array}$ & $8085,4053,2412$ \\
\hline$R_{\text {int }}$ & 0.033 \\
\hline$(\sin \theta / \lambda)_{\max }\left(\AA^{-1}\right)$ & 0.617 \\
\hline \multicolumn{2}{|l|}{ Refinement } \\
\hline$R\left[F^{2}>2 \sigma\left(F^{2}\right)\right], w R\left(F^{2}\right), S$ & $0.074,0.247,1.05$ \\
\hline No. of reflections & 4053 \\
\hline No. of parameters & 264 \\
\hline $\mathrm{H}$-atom treatment & $\mathrm{H}$-atom parameters constrained \\
\hline$\Delta \rho_{\max }, \Delta \rho_{\min }\left(\mathrm{e} \AA^{-3}\right)$ & $0.43,-0.34$ \\
\hline
\end{tabular}

Computer programs: CrysAlis PRO (Oxford Diffraction,2010), SHELXS97 and SHELXL97 (Sheldrick, 2008), ORTEP-3 for Windows (Farrugia, 2012) and PLATON (Spek, 2009). 
collected by filtration, washed with cold water and recrystallized at room temperature from a mixture of methanol and $N, N$-dimethyl formamide $(1: 1 v / v)$ giving yellow block-like crystals [m.p. $449 \mathrm{~K}$ ]

\section{Refinement}

Crystal data, data collection and structure refinement details are summarized in Table 2.

\section{Acknowledgements}

RK acknowledges the Indian Council of Medical Research, New Delhi, for financial support under Research Project No. $\mathrm{BIC} / 12$ (14)/2012. K is thankful to the UGC for funding under Project No. MRP-MAJOR-PHYS-2013-26952 (RP-88). SNK acknowledges the Department of Chemistry, ShriMadhwa-
Vadiraja Institute of Technology, Bantakal (VTU Belgam), for providing research facilities.

\section{References}

Farrugia, L. J. (2012). J. Appl. Cryst. 45, 849-854.

Mohamed, M. S., Mahmoud, R. K., Sayad, A. I. \& El-Araby, M. E. (2012). J. Med. Chem. 2, 24-29.

Oxford Diffraction (2010). CrysAlis PRO. Oxford Diffraction Ltd, Yarnton, England.

Patel, A., Bari, S., Talele, G., Patel, J. \& Sarangapani, M. (2006). Iran. J. Pharm. Res. 4, 249-254.

Sheldrick, G. M. (2008). Acta Cryst. A64, 112-122.

Spek, A. L. (2009). Acta Cryst. D65, 148-155.

Suhasini, G. E., Nirmala, M., Varalakshmi, Giri, A., Solomon, B. \& Sahitha, G. (2014). Int. J. Pharm. 4, 241-246.

Suthakaran, R., Kavimani, S., Venkaiaiah, P. \& Suganthi, K. (2008). Rasayan J. Chem. 1, 22-29. 


\section{full crystallographic data}

IUCrData (2016). 1, x160587 [doi:10.1107/S2414314616005873]

(4Z)-2-Phenyl-1-\{(E)-[4-(propan-2-yl)benzylidene]amino\}-4-[(thiophen-2yl)methylidene]-1 $H$-imidazol-5(4H)-one

Kamni, Vikram D. Singh, K. N. Subbulakshmi, B. Narayana, B. K. Sarojini, Sumati Anthal and Rajni Kant

(4Z)-2-Phenyl-1-\{(E)-[4-(propan-2-yl)benzylidene]amino\}-4-[(thiophen-2-yl)methylidene]-1 $H$-imidazol-5(4H)one

Crystal data

$\mathrm{C}_{24} \mathrm{H}_{21} \mathrm{~N}_{3} \mathrm{OS}$

$M_{r}=399.50$

Monoclinic, $P 2_{1} / c$

Hall symbol: $-\mathrm{P} 2 \mathrm{ybc}$

$a=15.8392(13) \AA$

$b=12.9075(10) \AA$

$c=10.3997(8) \AA$

$\beta=103.354(8)^{\circ}$

$V=2068.7(3) \AA^{3}$

$Z=4$

Data collection

Oxford Diffraction Xcalibur, Sapphire3 diffractometer

Radiation source: fine-focus sealed tube Graphite monochromator

Detector resolution: 16.1049 pixels $\mathrm{mm}^{-1}$

$\omega$ scans

Absorption correction: multi-scan

(CrysAlis PRO; Oxford Diffraction, 2010

$T_{\min }=0.636, T_{\max }=1.000$

\section{Refinement}

Refinement on $F^{2}$

Least-squares matrix: full

$R\left[F^{2}>2 \sigma\left(F^{2}\right)\right]=0.074$

$w R\left(F^{2}\right)=0.247$

$S=1.05$

4053 reflections

264 parameters

0 restraints

Primary atom site location: structure-invariant direct methods
$F(000)=840$

$D_{\mathrm{x}}=1.283 \mathrm{Mg} \mathrm{m}^{-3}$

Mo $K \alpha$ radiation, $\lambda=0.71073 \AA$

Cell parameters from 1816 reflections

$\theta=4.1-25.6^{\circ}$

$\mu=0.18 \mathrm{~mm}^{-1}$

$T=293 \mathrm{~K}$

Block, yellow

$0.30 \times 0.20 \times 0.20 \mathrm{~mm}$

8085 measured reflections

4053 independent reflections

2412 reflections with $I>2 \sigma(I)$

$R_{\text {int }}=0.033$

$\theta_{\text {max }}=26.0^{\circ}, \theta_{\min }=3.4^{\circ}$

$h=-19 \rightarrow 19$

$k=-15 \rightarrow 15$

$l=-10 \rightarrow 12$

Secondary atom site location: difference Fourier map

Hydrogen site location: inferred from neighbouring sites

$\mathrm{H}$-atom parameters constrained

$w=1 /\left[\sigma^{2}\left(F_{\mathrm{o}}^{2}\right)+(0.1066 P)^{2}+1.5103 P\right]$

where $P=\left(F_{\mathrm{o}}{ }^{2}+2 F_{\mathrm{c}}{ }^{2}\right) / 3$

$(\Delta / \sigma)_{\max }=0.001$

$\Delta \rho_{\max }=0.43 \mathrm{e} \AA^{-3}$

$\Delta \rho_{\min }=-0.34$ e $\AA^{-3}$ 


\section{Special details}

Experimental. CrysAlisPro, Oxford Diffraction Ltd., Version 1.171.34.40 (release 27-08-2010 CrysAlis171 .NET) (compiled Aug 27 2010,11:50:40) Empirical absorption correction using spherical harmonics, implemented in SCALE3 ABSPACK scaling algorithm.

Geometry. All esds (except the esd in the dihedral angle between two 1.s. planes) are estimated using the full covariance matrix. The cell esds are taken into account individually in the estimation of esds in distances, angles and torsion angles; correlations between esds in cell parameters are only used when they are defined by crystal symmetry. An approximate (isotropic) treatment of cell esds is used for estimating esds involving l.s. planes.

Refinement. Refinement of $\mathrm{F}^{2}$ against ALL reflections. The weighted R-factor $\mathrm{wR}$ and goodness of fit $\mathrm{S}$ are based on $\mathrm{F}^{2}$, conventional R-factors $R$ are based on $F$, with $F$ set to zero for negative $F^{2}$. The threshold expression of $F^{2}>2 \operatorname{sigma}\left(F^{2}\right)$ is used only for calculating R-factors(gt) etc. and is not relevant to the choice of reflections for refinement. R-factors based on $\mathrm{F}^{2}$ are statistically about twice as large as those based on F, and R- factors based on ALL data will be even larger.

Fractional atomic coordinates and isotropic or equivalent isotropic displacement parameters $\left(\AA^{2}\right)$

\begin{tabular}{|c|c|c|c|c|}
\hline & $x$ & $y$ & $z$ & $U_{\text {iso }} * / U_{\text {eq }}$ \\
\hline $\mathrm{S} 1$ & $0.48932(8)$ & $0.61536(9)$ & $0.93165(13)$ & $0.0810(5)$ \\
\hline N3 & $0.2515(2)$ & $0.9237(3)$ & $1.1960(3)$ & $0.0596(8)$ \\
\hline N1 & 0.37039 (19) & $0.7338(2)$ & $1.0619(3)$ & $0.0565(8)$ \\
\hline N2 & 0.31414 (19) & $0.8713(2)$ & $1.1460(3)$ & $0.0586(8)$ \\
\hline $\mathrm{O} 1$ & $0.3822(2)$ & $1.0076(2)$ & $1.0621(3)$ & $0.0852(10)$ \\
\hline $\mathrm{C} 9$ & $0.2618(2)$ & $0.6909(3)$ & $1.1846(4)$ & $0.0521(9)$ \\
\hline $\mathrm{C} 17$ & 0.1300 & $1.0434(3)$ & $1.2985(4)$ & $0.0661(11)$ \\
\hline H17 & 0.1079 & 0.9851 & 1.2497 & $0.079 *$ \\
\hline $\mathrm{C} 8$ & $0.3151(2)$ & $0.7636(3)$ & $1.1301(4)$ & $0.0535(9)$ \\
\hline $\mathrm{C} 5$ & $0.4681(2)$ & $0.8271(3)$ & $0.9533(4)$ & $0.0639(10)$ \\
\hline H5 & 0.4867 & 0.8930 & 0.9363 & $0.077^{*}$ \\
\hline $\mathrm{C} 16$ & $0.2157(2)$ & $1.0712(3)$ & $1.3090(4)$ & $0.0557(9)$ \\
\hline C6 & $0.4073(2)$ & 0.8230 & $1.0243(4)$ & $0.0601(10)$ \\
\hline $\mathrm{C} 14$ & $0.2251(3)$ & 0.7125 & $1.2905(4)$ & $0.0634(10)$ \\
\hline H14 & 0.2293 & 0.7789 & 1.3263 & $0.076^{*}$ \\
\hline $\mathrm{C} 4$ & $0.5077(2)$ & 0.7429 & $0.9007(4)$ & $0.0602(10)$ \\
\hline $\mathrm{C} 15$ & $0.2759(3)$ & $1.0096(3)$ & $1.2529(4)$ & $0.0606(10)$ \\
\hline H15 & 0.3322 & 1.0331 & 1.2588 & $0.073^{*}$ \\
\hline C19 & 0.1081 & $1.1886(4)$ & $1.4336(5)$ & $0.0773(13)$ \\
\hline $\mathrm{C} 7$ & $0.3709(3)$ & $0.9153(3)$ & $1.0755(4)$ & $0.0646(11)$ \\
\hline $\mathrm{C} 13$ & 0.1821 & $0.6353(4)$ & $1.3432(4)$ & $0.0736(12)$ \\
\hline H13 & 0.1591 & 0.6496 & 1.4158 & $0.088^{*}$ \\
\hline $\mathrm{C} 10$ & 0.2515 & $0.5916(3)$ & $1.1311(5)$ & $0.0702(12)$ \\
\hline H10 & 0.2744 & 0.5763 & 1.0587 & $0.084^{*}$ \\
\hline $\mathrm{C} 21$ & $0.2456(3)$ & $1.1606(3)$ & $1.3806(4)$ & 0.0687 (11) \\
\hline $\mathrm{H} 21$ & 0.3025 & 1.1822 & 1.3874 & $0.082 *$ \\
\hline $\mathrm{C} 18$ & $0.0771(3)$ & $1.1004(4)$ & $1.3589(5)$ & 0.0765 (13) \\
\hline H18 & 0.0197 & 1.0802 & 1.3502 & $0.092 *$ \\
\hline $\mathrm{C} 3$ & 0.5704 & $0.7537(3)$ & $0.8164(4)$ & $0.0612(10)$ \\
\hline $\mathrm{H} 3$ & 0.5913 & 0.8153 & 0.7894 & $0.073 *$ \\
\hline $\mathrm{C} 20$ & $0.1924(3)$ & $1.2173(3)$ & $1.4413(5)$ & 0.0796 (14) \\
\hline $\mathrm{H} 20$ & 0.2140 & 1.2765 & 1.4886 & $0.096^{*}$ \\
\hline $\mathrm{C} 1$ & $0.5561(3)$ & $0.5752(4)$ & $0.8383(5)$ & 0.0809 (13) \\
\hline
\end{tabular}




$\begin{array}{lllll}\mathrm{H} 1 & 0.5669 & 0.5056 & 0.8249 & 0.097^{*} \\ \mathrm{C} 11 & 0.2084(3) & 0.5161(4) & 1.1823(5) & 0.0862(15) \\ \mathrm{H} 11 & 0.2028 & 0.4501 & 1.1455 & 0.103^{*} \\ \mathrm{C} 2 & 0.5925(3) & 0.6525(4) & 0.7848(5) & 0.0877(15) \\ \mathrm{H} 2 & 0.6302 & 0.6403 & 0.7300 & 0.105^{*} \\ \mathrm{C} 22 & 0.0515(4) & 1.2478(4) & 1.5091(7) & 0.112(2) \\ \mathrm{H} 22 & 0.0963 & 1.2742 & 1.5832 & 0.135^{*} \\ \mathrm{C} 12 & 0.1736(3) & 0.5381(4) & 1.2884(5) & 0.0834(14) \\ \mathrm{H} 12 & 0.1441 & 0.4870 & 1.3233 & 0.100^{*} \\ \mathrm{C} 23 & 0.0029(5) & 1.1847(5) & 1.5790(6) & 0.137(3) \\ \mathrm{H} 23 \mathrm{~A} & -0.0227 & 1.2276 & 1.6352 & 0.206^{*} \\ \mathrm{H} 23 \mathrm{~B} & 0.0408 & 1.1350 & 1.6318 & 0.206^{*} \\ \mathrm{H} 23 \mathrm{C} & -0.0421 & 1.1492 & 1.5169 & 0.206^{*} \\ \mathrm{C} 24 & 0.0168(5) & 1.3427(5) & 1.4508(7) & 0.138(3) \\ \mathrm{H} 24 \mathrm{~A} & -0.0288 & 1.3286 & 1.3744 & 0.207^{*} \\ \mathrm{H} 24 \mathrm{~B} & 0.0617 & 1.3818 & 1.4251 & 0.207^{*} \\ \mathrm{H} 24 \mathrm{C} & -0.0060 & 1.3818 & 1.5136 & 0.207^{*} \\ \end{array}$

Atomic displacement parameters $\left(\AA^{2}\right)$

\begin{tabular}{lllllll}
\hline & $U^{11}$ & $U^{22}$ & $U^{33}$ & $U^{12}$ & $U^{13}$ & $U^{23}$ \\
\hline S1 & $0.0782(8)$ & $0.0620(8)$ & $0.1081(10)$ & $-0.0024(6)$ & $0.0322(7)$ & $-0.0085(6)$ \\
N3 & $0.0539(18)$ & $0.0530(19)$ & $0.076(2)$ & $0.0012(15)$ & $0.0237(16)$ & $-0.0084(16)$ \\
N1 & $0.0487(17)$ & $0.0492(18)$ & $0.074(2)$ & $-0.0024(14)$ & $0.0204(16)$ & $-0.0050(16)$ \\
N2 & $0.0534(18)$ & $0.0491(19)$ & $0.079(2)$ & $-0.0051(14)$ & $0.0273(16)$ & $-0.0111(16)$ \\
O1 & $0.085(2)$ & $0.0509(19)$ & $0.135(3)$ & $-0.0133(15)$ & $0.056(2)$ & $-0.0143(18)$ \\
C9 & $0.0396(18)$ & $0.053(2)$ & $0.062(2)$ & $-0.0018(16)$ & $0.0080(16)$ & $-0.0053(18)$ \\
C17 & $0.060(2)$ & $0.055(2)$ & $0.085(3)$ & $-0.0010(19)$ & $0.020(2)$ & $-0.009(2)$ \\
C8 & $0.0459(19)$ & $0.051(2)$ & $0.063(2)$ & $-0.0013(16)$ & $0.0104(17)$ & $-0.0071(18)$ \\
C5 & $0.056(2)$ & $0.054(2)$ & $0.085(3)$ & $-0.0081(18)$ & $0.025(2)$ & $-0.007(2)$ \\
C16 & $0.057(2)$ & $0.048(2)$ & $0.065(2)$ & $0.0024(17)$ & $0.0205(18)$ & $0.0003(18)$ \\
C6 & $0.048(2)$ & $0.057(2)$ & $0.077(3)$ & $-0.0055(18)$ & $0.0181(19)$ & $-0.010(2)$ \\
C14 & $0.066(2)$ & $0.057(2)$ & $0.069(2)$ & $0.0005(19)$ & $0.019(2)$ & $-0.0049(19)$ \\
C4 & $0.054(2)$ & $0.057(2)$ & $0.071(2)$ & $-0.0005(18)$ & $0.0173(19)$ & $-0.002(2)$ \\
C15 & $0.055(2)$ & $0.052(2)$ & $0.080(3)$ & $-0.0061(18)$ & $0.025(2)$ & $-0.009(2)$ \\
C19 & $0.089(3)$ & $0.057(3)$ & $0.099(3)$ & $0.016(2)$ & $0.049(3)$ & $0.010(2)$ \\
C7 & $0.056(2)$ & $0.054(3)$ & $0.087(3)$ & $-0.0052(18)$ & $0.024(2)$ & $-0.009(2)$ \\
C13 & $0.064(3)$ & $0.082(3)$ & $0.081(3)$ & $0.002(2)$ & $0.029(2)$ & $0.009(3)$ \\
C10 & $0.065(3)$ & $0.060(3)$ & $0.089(3)$ & $-0.007(2)$ & $0.025(2)$ & $-0.014(2)$ \\
C21 & $0.068(3)$ & $0.055(2)$ & $0.089(3)$ & $-0.008(2)$ & $0.031(2)$ & $-0.009(2)$ \\
C18 & $0.057(2)$ & $0.072(3)$ & $0.107(3)$ & $0.005(2)$ & $0.033(2)$ & $0.004(3)$ \\
C3 & $0.068(2)$ & $0.048(2)$ & $0.073(2)$ & $0.0073(19)$ & $0.026(2)$ & $-0.0071(19)$ \\
C20 & $0.099(4)$ & $0.052(3)$ & $0.101(3)$ & $0.000(2)$ & $0.050(3)$ & $-0.010(2)$ \\
C1 & $0.087(3)$ & $0.067(3)$ & $0.093(3)$ & $0.008(3)$ & $0.027(3)$ & $-0.017(3)$ \\
C11 & $0.085(3)$ & $0.056(3)$ & $0.126(4)$ & $-0.017(2)$ & $0.044(3)$ & $-0.012(3)$ \\
C2 & $0.085(3)$ & $0.101(4)$ & $0.085(3)$ & $0.005(3)$ & $0.037(3)$ & $0.000(3)$ \\
C22 & $0.131(5)$ & $0.075(4)$ & $0.160(5)$ & $0.017(3)$ & $0.094(5)$ & $0.000(4)$ \\
C12 & $0.068(3)$ & $0.072(3)$ & $0.115(4)$ & $-0.011(2)$ & $0.031(3)$ & $0.014(3)$
\end{tabular}




$\begin{array}{lllllll}\mathrm{C} 23 & 0.188(7) & 0.120(5) & 0.145(5) & 0.066(5) & 0.123(5) & 0.049(4) \\ \mathrm{C} 24 & 0.196(7) & 0.094(4) & 0.164(6) & 0.077(5) & 0.126(6) & 0.050(4)\end{array}$

Geometric parameters $(\AA, \stackrel{o}{)}$

\begin{tabular}{|c|c|c|c|}
\hline $\mathrm{S} 1-\mathrm{C} 1$ & $1.675(5)$ & $\mathrm{C} 19-\mathrm{C} 22$ & $1.526(6)$ \\
\hline $\mathrm{S} 1-\mathrm{C} 4$ & $1.715(4)$ & $\mathrm{C} 13-\mathrm{C} 12$ & $1.372(7)$ \\
\hline N3-C15 & $1.274(5)$ & $\mathrm{C} 13-\mathrm{H} 13$ & 0.9300 \\
\hline $\mathrm{N} 3-\mathrm{N} 2$ & $1.397(4)$ & $\mathrm{C} 10-\mathrm{C} 11$ & $1.366(6)$ \\
\hline $\mathrm{N} 1-\mathrm{C} 8$ & $1.306(4)$ & $\mathrm{C} 10-\mathrm{H} 10$ & 0.9300 \\
\hline $\mathrm{N} 1-\mathrm{C} 6$ & $1.388(5)$ & $\mathrm{C} 21-\mathrm{C} 20$ & $1.376(6)$ \\
\hline $\mathrm{N} 2-\mathrm{C} 8$ & $1.400(5)$ & $\mathrm{C} 21-\mathrm{H} 21$ & 0.9300 \\
\hline $\mathrm{N} 2-\mathrm{C} 7$ & $1.405(5)$ & $\mathrm{C} 18-\mathrm{H} 18$ & 0.9300 \\
\hline $\mathrm{O} 1-\mathrm{C} 7$ & $1.218(5)$ & $\mathrm{C} 3-\mathrm{C} 2$ & $1.410(6)$ \\
\hline $\mathrm{C} 9-\mathrm{C} 14$ & $1.387(5)$ & $\mathrm{C} 3-\mathrm{H} 3$ & 0.9300 \\
\hline $\mathrm{C} 9-\mathrm{C} 10$ & $1.392(5)$ & $\mathrm{C} 20-\mathrm{H} 20$ & 0.9300 \\
\hline $\mathrm{C} 9-\mathrm{C} 8$ & $1.461(5)$ & $\mathrm{C} 1-\mathrm{C} 2$ & $1.336(7)$ \\
\hline $\mathrm{C} 17-\mathrm{C} 18$ & $1.372(6)$ & $\mathrm{C} 1-\mathrm{H} 1$ & 0.9300 \\
\hline $\mathrm{C} 17-\mathrm{C} 16$ & $1.384(5)$ & $\mathrm{C} 11-\mathrm{C} 12$ & $1.372(7)$ \\
\hline C17-H17 & 0.9300 & $\mathrm{C} 11-\mathrm{H} 11$ & 0.9300 \\
\hline $\mathrm{C} 5-\mathrm{C} 6$ & $1.343(5)$ & $\mathrm{C} 2-\mathrm{H} 2$ & 0.9300 \\
\hline $\mathrm{C} 5-\mathrm{C} 4$ & $1.426(6)$ & $\mathrm{C} 22-\mathrm{C} 24$ & $1.419(7)$ \\
\hline $\mathrm{C} 5-\mathrm{H} 5$ & 0.9300 & $\mathrm{C} 22-\mathrm{C} 23$ & $1.429(7)$ \\
\hline $\mathrm{C} 16-\mathrm{C} 21$ & $1.395(5)$ & $\mathrm{C} 22-\mathrm{H} 22$ & 0.9800 \\
\hline $\mathrm{C} 16-\mathrm{C} 15$ & $1.463(5)$ & $\mathrm{C} 12-\mathrm{H} 12$ & 0.9300 \\
\hline $\mathrm{C} 6-\mathrm{C} 7$ & $1.476(6)$ & $\mathrm{C} 23-\mathrm{H} 23 \mathrm{~A}$ & 0.9600 \\
\hline $\mathrm{C} 14-\mathrm{C} 13$ & $1.389(6)$ & $\mathrm{C} 23-\mathrm{H} 23 \mathrm{~B}$ & 0.9600 \\
\hline C14-H14 & 0.9300 & $\mathrm{C} 23-\mathrm{H} 23 \mathrm{C}$ & 0.9600 \\
\hline $\mathrm{C} 4-\mathrm{C} 3$ & $1.474(5)$ & $\mathrm{C} 24-\mathrm{H} 24 \mathrm{~A}$ & 0.9600 \\
\hline $\mathrm{C} 15-\mathrm{H} 15$ & 0.9300 & $\mathrm{C} 24-\mathrm{H} 24 \mathrm{~B}$ & 0.9600 \\
\hline $\mathrm{C} 19-\mathrm{C} 20$ & $1.369(6)$ & $\mathrm{C} 24-\mathrm{H} 24 \mathrm{C}$ & 0.9600 \\
\hline $\mathrm{C} 19-\mathrm{C} 18$ & $1.402(6)$ & & \\
\hline $\mathrm{C} 1-\mathrm{S} 1-\mathrm{C} 4$ & $91.8(2)$ & $\mathrm{C} 9-\mathrm{C} 10-\mathrm{H} 10$ & 119.2 \\
\hline $\mathrm{C} 15-\mathrm{N} 3-\mathrm{N} 2$ & $115.4(3)$ & $\mathrm{C} 20-\mathrm{C} 21-\mathrm{C} 16$ & $121.1(4)$ \\
\hline $\mathrm{C} 8-\mathrm{N} 1-\mathrm{C} 6$ & $106.8(3)$ & $\mathrm{C} 20-\mathrm{C} 21-\mathrm{H} 21$ & 119.5 \\
\hline $\mathrm{N} 3-\mathrm{N} 2-\mathrm{C} 8$ & $123.5(3)$ & $\mathrm{C} 16-\mathrm{C} 21-\mathrm{H} 21$ & 119.5 \\
\hline $\mathrm{N} 3-\mathrm{N} 2-\mathrm{C} 7$ & $126.4(3)$ & $\mathrm{C} 17-\mathrm{C} 18-\mathrm{C} 19$ & $121.1(4)$ \\
\hline $\mathrm{C} 8-\mathrm{N} 2-\mathrm{C} 7$ & $108.4(3)$ & $\mathrm{C} 17-\mathrm{C} 18-\mathrm{H} 18$ & 119.4 \\
\hline $\mathrm{C} 14-\mathrm{C} 9-\mathrm{C} 10$ & $118.1(4)$ & $\mathrm{C} 19-\mathrm{C} 18-\mathrm{H} 18$ & 119.4 \\
\hline $\mathrm{C} 14-\mathrm{C} 9-\mathrm{C} 8$ & $124.3(4)$ & $\mathrm{C} 2-\mathrm{C} 3-\mathrm{C} 4$ & $106.8(4)$ \\
\hline $\mathrm{C} 10-\mathrm{C} 9-\mathrm{C} 8$ & $117.5(3)$ & $\mathrm{C} 2-\mathrm{C} 3-\mathrm{H} 3$ & 126.6 \\
\hline $\mathrm{C} 18-\mathrm{C} 17-\mathrm{C} 16$ & $121.1(4)$ & $\mathrm{C} 4-\mathrm{C} 3-\mathrm{H} 3$ & 126.6 \\
\hline $\mathrm{C} 18-\mathrm{C} 17-\mathrm{H} 17$ & 119.5 & $\mathrm{C} 19-\mathrm{C} 20-\mathrm{C} 21$ & $121.4(4)$ \\
\hline $\mathrm{C} 16-\mathrm{C} 17-\mathrm{H} 17$ & 119.5 & $\mathrm{C} 19-\mathrm{C} 20-\mathrm{H} 20$ & 119.3 \\
\hline $\mathrm{N} 1-\mathrm{C} 8-\mathrm{N} 2$ & $112.5(3)$ & $\mathrm{C} 21-\mathrm{C} 20-\mathrm{H} 20$ & 119.3 \\
\hline $\mathrm{N} 1-\mathrm{C} 8-\mathrm{C} 9$ & $122.8(3)$ & $\mathrm{C} 2-\mathrm{C} 1-\mathrm{S} 1$ & $113.6(4)$ \\
\hline $\mathrm{N} 2-\mathrm{C} 8-\mathrm{C} 9$ & $124.7(3)$ & $\mathrm{C} 2-\mathrm{C} 1-\mathrm{H} 1$ & 123.2 \\
\hline
\end{tabular}


C6- 5 - 4

$\mathrm{C} 6-\mathrm{C} 5-\mathrm{H} 5$

$\mathrm{C} 4-\mathrm{C} 5-\mathrm{H} 5$

$\mathrm{C} 17-\mathrm{C} 16-\mathrm{C} 21$

$\mathrm{C} 17-\mathrm{C} 16-\mathrm{C} 15$

$\mathrm{C} 21-\mathrm{C} 16-\mathrm{C} 15$

$\mathrm{C} 5-\mathrm{C} 6-\mathrm{N} 1$

$\mathrm{C} 5-\mathrm{C} 6-\mathrm{C} 7$

$\mathrm{N} 1-\mathrm{C} 6-\mathrm{C} 7$

C9-C14-C13

C9- $\mathrm{C} 14-\mathrm{H} 14$

$\mathrm{C} 13-\mathrm{C} 14-\mathrm{H} 14$

$\mathrm{C} 5-\mathrm{C} 4-\mathrm{C} 3$

$\mathrm{C} 5-\mathrm{C} 4-\mathrm{S} 1$

$\mathrm{C} 3-\mathrm{C} 4-\mathrm{S} 1$

N3-C15-C16

N3- C15-H15

$\mathrm{C} 16-\mathrm{C} 15-\mathrm{H} 15$

$\mathrm{C} 20-\mathrm{C} 19-\mathrm{C} 18$

$\mathrm{C} 20-\mathrm{C} 19-\mathrm{C} 22$

$\mathrm{C} 18-\mathrm{C} 19-\mathrm{C} 22$

$\mathrm{O} 1-\mathrm{C} 7-\mathrm{N} 2$

$\mathrm{O} 1-\mathrm{C} 7-\mathrm{C} 6$

$\mathrm{N} 2-\mathrm{C} 7-\mathrm{C} 6$

$\mathrm{C} 12-\mathrm{C} 13-\mathrm{C} 14$

C12-C13-H13

$\mathrm{C} 14-\mathrm{C} 13-\mathrm{H} 13$

$\mathrm{C} 11-\mathrm{C} 10-\mathrm{C} 9$

$\mathrm{C} 11-\mathrm{C} 10-\mathrm{H} 10$

$\mathrm{C} 15-\mathrm{N} 3-\mathrm{N} 2-\mathrm{C} 8$

$\mathrm{C} 15-\mathrm{N} 3-\mathrm{N} 2-\mathrm{C} 7$

$\mathrm{C} 6-\mathrm{N} 1-\mathrm{C} 8-\mathrm{N} 2$

$\mathrm{C} 6-\mathrm{N} 1-\mathrm{C} 8-\mathrm{C} 9$

$\mathrm{N} 3-\mathrm{N} 2-\mathrm{C} 8-\mathrm{N} 1$

$\mathrm{C} 7-\mathrm{N} 2-\mathrm{C} 8-\mathrm{N} 1$

$\mathrm{N} 3-\mathrm{N} 2-\mathrm{C} 8-\mathrm{C} 9$

$\mathrm{C} 7-\mathrm{N} 2-\mathrm{C} 8-\mathrm{C} 9$

$\mathrm{C} 14-\mathrm{C} 9-\mathrm{C} 8-\mathrm{N} 1$

$\mathrm{C} 10-\mathrm{C} 9-\mathrm{C} 8-\mathrm{N} 1$

$\mathrm{C} 14-\mathrm{C} 9-\mathrm{C} 8-\mathrm{N} 2$

$\mathrm{C} 10-\mathrm{C} 9-\mathrm{C} 8-\mathrm{N} 2$

$\mathrm{C} 18-\mathrm{C} 17-\mathrm{C} 16-\mathrm{C} 21$

$\mathrm{C} 18-\mathrm{C} 17-\mathrm{C} 16-\mathrm{C} 15$

$\mathrm{C} 4-\mathrm{C} 5-\mathrm{C} 6-\mathrm{N} 1$

$\mathrm{C} 4-\mathrm{C} 5-\mathrm{C} 6-\mathrm{C} 7$

$\mathrm{C} 8-\mathrm{N} 1-\mathrm{C} 6-\mathrm{C} 5$

$\mathrm{C} 8-\mathrm{N} 1-\mathrm{C} 6-\mathrm{C} 7$
$128.0(4)$

116.0

116.0

117.6 (3)

123.0 (4)

119.4 (3)

126.2 (4)

123.8 (4)

110.0 (3)

120.1 (4)

119.9

119.9

124.9 (4)

123.4 (3)

$111.6(3)$

120.2 (3)

119.9

119.9

117.7 (4)

$121.0(5)$

$121.3(5)$

125.8 (4)

132.0 (4)

102.2 (3)

120.2 (4)

119.9

119.9

$121.6(4)$

119.2

153.1 (4)

$-43.6(5)$

-2.2 (4)

178.7 (3)

169.3 (3)

3.4 (4)

$-11.6(6)$

-177.6 (4)

157.6 (4)

$-18.7(5)$

$-21.4(6)$

162.3 (4)

$-1.5(6)$

176.3 (4)

$0.3(7)$

179.4 (4)

179.5 (4)

0.3 (4)
$\mathrm{S} 1-\mathrm{C} 1-\mathrm{H} 1$

$\mathrm{C} 10-\mathrm{C} 11-\mathrm{C} 12$

$\mathrm{C} 10-\mathrm{C} 11-\mathrm{H} 11$

C12-C11-H11

$\mathrm{C} 1-\mathrm{C} 2-\mathrm{C} 3$

$\mathrm{C} 1-\mathrm{C} 2-\mathrm{H} 2$

$\mathrm{C} 3-\mathrm{C} 2-\mathrm{H} 2$

$\mathrm{C} 24-\mathrm{C} 22-\mathrm{C} 23$

$\mathrm{C} 24-\mathrm{C} 22-\mathrm{C} 19$

$\mathrm{C} 23-\mathrm{C} 22-\mathrm{C} 19$

$\mathrm{C} 24-\mathrm{C} 22-\mathrm{H} 22$

$\mathrm{C} 23-\mathrm{C} 22-\mathrm{H} 22$

$\mathrm{C} 19-\mathrm{C} 22-\mathrm{H} 22$

C13-C12-C11

$\mathrm{C} 13-\mathrm{C} 12-\mathrm{H} 12$

$\mathrm{C} 11-\mathrm{C} 12-\mathrm{H} 12$

$\mathrm{C} 22-\mathrm{C} 23-\mathrm{H} 23 \mathrm{~A}$

$\mathrm{C} 22-\mathrm{C} 23-\mathrm{H} 23 \mathrm{~B}$

$\mathrm{H} 23 \mathrm{~A}-\mathrm{C} 23-\mathrm{H} 23 \mathrm{~B}$

$\mathrm{C} 22-\mathrm{C} 23-\mathrm{H} 23 \mathrm{C}$

$\mathrm{H} 23 \mathrm{~A}-\mathrm{C} 23-\mathrm{H} 23 \mathrm{C}$

$\mathrm{H} 23 \mathrm{~B}-\mathrm{C} 23-\mathrm{H} 23 \mathrm{C}$

$\mathrm{C} 22-\mathrm{C} 24-\mathrm{H} 24 \mathrm{~A}$

$\mathrm{C} 22-\mathrm{C} 24-\mathrm{H} 24 \mathrm{~B}$

$\mathrm{H} 24 \mathrm{~A}-\mathrm{C} 24-\mathrm{H} 24 \mathrm{~B}$

$\mathrm{C} 22-\mathrm{C} 24-\mathrm{H} 24 \mathrm{C}$

$\mathrm{H} 24 \mathrm{~A}-\mathrm{C} 24-\mathrm{H} 24 \mathrm{C}$

$\mathrm{H} 24 \mathrm{~B}-\mathrm{C} 24-\mathrm{H} 24 \mathrm{C}$

$\mathrm{N} 3-\mathrm{N} 2-\mathrm{C} 7-\mathrm{C} 6$

$\mathrm{C} 8-\mathrm{N} 2-\mathrm{C} 7-\mathrm{C} 6$

$\mathrm{C} 5-\mathrm{C} 6-\mathrm{C} 7-\mathrm{O} 1$

$\mathrm{N} 1-\mathrm{C} 6-\mathrm{C} 7-\mathrm{O} 1$

$\mathrm{C} 5-\mathrm{C} 6-\mathrm{C} 7-\mathrm{N} 2$

$\mathrm{N} 1-\mathrm{C} 6-\mathrm{C} 7-\mathrm{N} 2$

$\mathrm{C} 9-\mathrm{C} 14-\mathrm{C} 13-\mathrm{C} 12$

$\mathrm{C} 14-\mathrm{C} 9-\mathrm{C} 10-\mathrm{C} 11$

$\mathrm{C} 8-\mathrm{C} 9-\mathrm{C} 10-\mathrm{C} 11$

$\mathrm{C} 17-\mathrm{C} 16-\mathrm{C} 21-\mathrm{C} 20$

$\mathrm{C} 15-\mathrm{C} 16-\mathrm{C} 21-\mathrm{C} 20$

$\mathrm{C} 16-\mathrm{C} 17-\mathrm{C} 18-\mathrm{C} 19$

$\mathrm{C} 20-\mathrm{C} 19-\mathrm{C} 18-\mathrm{C} 17$

$\mathrm{C} 22-\mathrm{C} 19-\mathrm{C} 18-\mathrm{C} 17$

$\mathrm{C} 5-\mathrm{C} 4-\mathrm{C} 3-\mathrm{C} 2$

$\mathrm{S} 1-\mathrm{C} 4-\mathrm{C} 3-\mathrm{C} 2$

$\mathrm{C} 18-\mathrm{C} 19-\mathrm{C} 20-\mathrm{C} 21$

$\mathrm{C} 22-\mathrm{C} 19-\mathrm{C} 20-\mathrm{C} 21$
123.2

119.7 (5)

120.2

120.2

116.1 (4)

121.9

121.9

120.5 (5)

115.4 (5)

115.2 (5)

100.1

100.1

100.1

120.3 (4)

119.9

119.9

109.5

109.5

109.5

109.5

109.5

109.5

109.5

109.5

109.5

109.5

109.5

109.5

-168.3 (3)

-2.9 (4)

3.8 (8)

-176.9 (5)

-177.6 (4)

1.7 (4)

-1.9 (6)

$-1.6(6)$

174.9 (4)

1.6 (6)

-176.2 (4)

$-0.2(7)$

$1.7(7)$

$-175.6(5)$

$-179.2(4)$

2.1 (4)

$-1.5(7)$

$175.8(5)$ 


\begin{tabular}{ll}
$\mathrm{C} 10-\mathrm{C} 9-\mathrm{C} 14-\mathrm{C} 13$ & $2.2(6)$ \\
$\mathrm{C} 8-\mathrm{C} 9-\mathrm{C} 14-\mathrm{C} 13$ & $-174.1(4)$ \\
$\mathrm{C} 6-\mathrm{C} 5-\mathrm{C} 4-\mathrm{C} 3$ & $176.0(4)$ \\
$\mathrm{C} 6-\mathrm{C} 5-\mathrm{C} 4-\mathrm{S} 1$ & $-5.4(6)$ \\
$\mathrm{C} 1-\mathrm{S} 1-\mathrm{C} 4-\mathrm{C} 5$ & $179.8(4)$ \\
$\mathrm{C} 1-\mathrm{S} 1-\mathrm{C} 4-\mathrm{C} 3$ & $-1.4(3)$ \\
$\mathrm{N} 2-\mathrm{N} 3-\mathrm{C} 15-\mathrm{C} 16$ & $180.0(3)$ \\
$\mathrm{C} 17-\mathrm{C} 16-\mathrm{C} 15-\mathrm{N} 3$ & $-3.1(6)$ \\
$\mathrm{C} 21-\mathrm{C} 16-\mathrm{C} 15-\mathrm{N} 3$ & $174.6(4)$ \\
$\mathrm{N} 3-\mathrm{N} 2-\mathrm{C} 7-\mathrm{O} 1$ & $10.4(7)$ \\
$\mathrm{C} 8-\mathrm{N} 2-\mathrm{C} 7-\mathrm{O} 1$ & $175.8(4)$ \\
\hline
\end{tabular}

$\begin{array}{ll}\mathrm{C} 16-\mathrm{C} 21-\mathrm{C} 20-\mathrm{C} 19 & -0.2(7) \\ \mathrm{C} 4-\mathrm{S} 1-\mathrm{C} 1-\mathrm{C} 2 & 0.4(4) \\ \mathrm{C} 9-\mathrm{C} 10-\mathrm{C} 11-\mathrm{C} 12 & 0.6(7) \\ \mathrm{S} 1-\mathrm{C} 1-\mathrm{C} 2-\mathrm{C} 3 & 0.9(6) \\ \mathrm{C} 4-\mathrm{C} 3-\mathrm{C} 2-\mathrm{C} 1 & -1.9(6) \\ \mathrm{C} 20-\mathrm{C} 19-\mathrm{C} 22-\mathrm{C} 24 & 78.6(8) \\ \mathrm{C} 18-\mathrm{C} 19-\mathrm{C} 22-\mathrm{C} 24 & -104.2(7) \\ \mathrm{C} 20-\mathrm{C} 19-\mathrm{C} 22-\mathrm{C} 23 & -133.9(6) \\ \mathrm{C} 18-\mathrm{C} 19-\mathrm{C} 22-\mathrm{C} 23 & 43.3(8) \\ \mathrm{C} 14-\mathrm{C} 13-\mathrm{C} 12-\mathrm{C} 11 & 0.9(7) \\ \mathrm{C} 10-\mathrm{C} 11-\mathrm{C} 12-\mathrm{C} 13 & -0.2(8)\end{array}$

Hydrogen-bond geometry $\left(\AA,{ }^{\circ}\right)$

\begin{tabular}{lllll}
\hline$D-\mathrm{H} \cdots A$ & $D-\mathrm{H}$ & $\mathrm{H} \cdots A$ & $D \cdots A$ & $D-\mathrm{H} \cdots A$ \\
\hline $\mathrm{C} 14-\mathrm{H} 14 \cdots \mathrm{N} 3$ & 0.93 & 2.38 & $2.960(5)$ & 120 \\
$\mathrm{C} 5-\mathrm{H} 5 \cdots \mathrm{O} 1^{\mathrm{i}}$ & 0.93 & 2.44 & $3.221(5)$ & 142 \\
$\mathrm{C} 3-\mathrm{H} 3 \cdots \mathrm{O} 1^{\mathrm{i}}$ & 0.93 & 2.74 & $3.349(5)$ & 124 \\
\hline
\end{tabular}

Symmetry code: (i) $-x+1,-y+2,-z+2$. 